\section{Small-volume hypertonic saline/pentastarch improves ileal mucosal microcirculation in experimental peritonitis}

\author{
Abdelnasser Assadi, ${ }^{1}$ Olivier Desebbe, ${ }^{1,3}$ \\ Thomas Rimmelé,, ${ }^{1,2}$ Arnal Florence, ${ }^{1}$ \\ Joëlle Goudable, ${ }^{5}$ Dominique Chassard, \\ Bernard Allaouchiche ${ }^{1,2}$ \\ ${ }^{1}$ Inserm ERI 22, Agressions vasculaires- \\ Réponses tissulaires, Claude Bernard \\ University-Lyon 1; 'Department of \\ Anesthesiology and Intensive Care, \\ Edouard Herriot Hospital; ${ }^{3}$ Department \\ of Anesthesiology and Intensive Care, \\ Louis Pradel Hospital; ${ }^{4}$ Department of \\ Anesthesiology and Intensive Care, \\ Mére-enfant Hospital; ${ }^{5}$ Laboratory of \\ Biochemistry, Edouard Herriot hospital, \\ Lyon, France
}

\section{Abstract}

We compared the effects of hypertonic saline $7.2 \% / 6 \%$ hydroxyethyl starch (HSS-HES) and isotonic saline $0.9 \% / 6 \%$ hydroxyethyl starch (ISS-HES) on ileal microcirculatory blood flow (MBF) at the initial phase of septic shock. Pigs were anesthetized and mechanically ventilated. Catheters were inserted into right atrium, pulmonary artery, carotid artery, and portal vein for hemodynamic measurements and for blood sampling. Ileal mucosal and muscularis MBF was continuously measured by laser Doppler flowmetry (LDF). Septic shock was obtained 240 min after induction of fecal peritonitis; then animals were randomized to receive $10 \mathrm{~mL} \cdot \mathrm{kg}^{-1}$ during $10 \mathrm{~min}$ of either HSS-HES or ISS-HES. Systemic and microcirculatory blood flow as well as systemic metabolism were assessed. Fecal peritonitis promoted a hypodynamic septic shock, with significant reduction of mean arterial pressure (MAP) and cardiac index (CI). Ileal mucosal MBF (-34\%) and ileal muscularis MBF (-54\%) significantly diminished from baseline. Contrary to ISS-HES group, mucosal MBF significantly augmented after HSS-HES (+192\% at min 150 post-shock) despite low blood pressure. There was weak correlation with $\mathrm{CI}\left(\mathrm{r}^{2}=\right.$ $0.2, \mathrm{P}=0.01)$. Muscularis MBF didn't change. HSS-HES-treated animals had a significantly higher osmolarity and sodium concentration than ISS-HES group. Other variables did not change. Small-volume resuscitation with HSSHES, but not ISS-HES, improved ileal microcirculatory impairment in experimental peritonitis model of septic shock even when MAP was low. This beneficial microcirculatory effect could be valuable in the management of early severe sepsis.

\section{Introduction}

During conventional management of severe sepsis, both isotonic crystalloid and colloid have been used to restore systemic hemodynamic status, but the volume of isotonic fluid required to restore cardiocirculatory function is large and often promoting partial and transient benefits, which are especially poor at the splanchnic bed. ${ }^{1}$ Since 1980 , when hyperosmotic $7.5 \%$ sodium chloride $(\mathrm{NaCl})$ was first described as a small-volume resuscitation solution, ${ }^{2}$ many clinical and animal studies were published. Hemodynamic effects of hypertonic saline resuscitation in hemorrhagic shock are well documented. Hypertonic saline solution (HSS) promotes both systemic and microcirculatory blood flow improvement, ${ }^{3}$ reduces bacterial translocation and lung injury after hemorrhage, ${ }^{4,5}$ diminishes neutrophil rolling and adherence to endothelium and reduces in vivo vascular leakage, ${ }^{6}$ enhances intracellular killing of bacteria while attenuating receptor-mediated activation of proinflammatory cascades. ${ }^{7,8}$ Although experience with the use of hypertonic saline solutions in sepsis is limited; these interesting benefits were started to be applied in sepsis. ${ }^{9}$

In addition, although vascular volume expansion occurs rapidly after infusion of HSS, volume expansion is relatively transient unless an oncotically effective colloid (dextran or hydroxyethyl starch) is added in order to preserve the intravascular volume gain. ${ }^{10}$ Contrary to dextran, no study has evaluated the effects of hypertonic saline/hydroxyethyl starch (HSS-HES) and isotonic saline/ hydroxyethyl starch (ISS-HES) in initial treatment of septic shock. Thus, our aim was to compare HSS-HES with ISS-HES on systemic, regional and microcirculatory blood flow as well as systemic metabolism in experimental peritonitis model of septic shock.

\section{Materials and Methods}

\section{Animal preparation}

The animal ethic committee of the University of Claude-Bernard-Lyon 1, Lyon, France, approved the protocol. Fourteen domestic female pigs (weight, $25 \mathrm{~kg}$ ) were fasted overnight but were allowed free access to water. Pigs were sedated with intramuscular injection of ketamine (10mg. $\left.\mathrm{kg}^{-1}\right)$. Anesthesia was induced with propofol ( $\left.3 \mathrm{mg} \cdot \mathrm{kg}^{-1}\right)$, and was maintained with sevoflurane (MAC $=2)$, sufen-
Correspondence: Assadi Abdelnasser,

24, Rue des Girondins 69007 Lyon, France.

E-mail: assadinasser@yahoo.com

Key words: hydroxyethylstarch, hypertonic saline, laser Doppler flowmetry, microcirculation, septic shock, splanchnic perfusion.

Received for publication: 6 October 2011. Accepted for publication: 8 January 2012.

This work is licensed under a Creative Commons Attribution NonCommercial 3.0 License (CC BYNC 3.0).

(C) Copyright A. Assadi et al., 2012

Licensee PAGEPress srl, Italy

Infectious Disease Reports 2012; 4:e22

doi:10.4081/idr.2012.e22

tanyl $\left(1 \mu \mathrm{g} \cdot \mathrm{kg}^{-1} \cdot \mathrm{hr}^{-1}\right)$ and cisatracurium at doses of $0.3 \mathrm{mg} \cdot \mathrm{kg}^{-1}$ every $30 \mathrm{~min}$. After performing a tracheostomy, animals were mechanically ventilated with a $\mathrm{FIO}_{2}$ that maintained an arterial partial pressure of $\mathrm{O}_{2}\left(\mathrm{PaO}_{2}\right)$ above 80 torr, at a tidal volume of $8-10 \mathrm{~mL}^{\mathrm{kg}}{ }^{-1}$, and respiratory rate that maintained $\mathrm{PaCO}_{2}$ at $40 \pm 5$ torr. End-expiratory pressure was zero.

Catheters were inserted into right carotid artery, for continuous monitoring of MAP and for collecting blood samples, and into right atrium through left external jugular vein for fluid and drug administration. A 7-Fr thermister-tip catheter (Baxter Edwards Critical-Care, Ivrine, CA) was advanced into pulmonary artery through right external jugular vein to measure mean pulmonary artery pressure (MPAP, mmHg), CVP (mmHg), PCWP (mmHg) and cardiac index (CI, mL.min $\left.{ }^{-1} \cdot \mathrm{kg}^{-1}\right)$, and to draw mixed venous blood samples. Location of pulmonary arterial catheter tip was determined by observing the characteristic pressure trace on the monitor as it was advanced through right heart into pulmonary artery. The central venous temperature was monitored with a thermistor in the pulmonary artery. Temperature of the animal was maintained at $37.2( \pm) 0.8^{\circ} \mathrm{C}$ with a heating pad and heat lamps. During surgical preparation, animals were given a continuous infusion of $15-20$ $\mathrm{mL} . \mathrm{kg}^{-1} \cdot \mathrm{h}^{-1}$ lactated Ringer solution to maintain CVP and PCWP values at 6-8 $\mathrm{mmHg}$.

A midline laparotomy was performed. The spleen was removed to avoid autotransfusion during shock ${ }^{11}$ and a catheter was inserted into portal vein for blood sampling. ${ }^{12} 0.75$ g.kg ${ }^{1}$ of autologous feces was collected through a small incision of antimesenteric border of the ascending colon, to be used later to induce fecal peritonitis and septic shock. Bowel incision was then closed with continuous sutures. A $5 \mathrm{~cm}$ segment of distal ileum, $20 \mathrm{~cm}$ proxi- 
mal to ileocecal junction, was defined. A platform was placed above the abdomen and fixed to a ring stand on either side of the pig. This platform has mobile and adjustable twopieces. The isolated ileal segment was opened along its entire antimesenteric border and exteriorized on ventral midline of the animal. The cut edges of ileum were stitched to the two-pieces of a platform. The height of the platform above the ventral abdominal wall was adjusted, and the two-pieces were positioned in such a way that mucosa of opened bowel was well exposed, but the mesentery supplying the segment was not compressed. A Laser Doppler flowmetry (LDF) probe was suspended, and positioned perpendicularly on intestinal mucosa with help of special miniholder (Perimed $^{\circledR}$, Probe 407 with PH 07 Miniholder); by this way, LDF probe would generate minimal pressure on tissue surface and artefacts caused by respiratory movements and intestinal peristalsis would be eliminated (extract of LDF recording Figure 1). In the same way, another LDF probe was placed on ileal serosa with help of a special miniholder (Perimed ${ }^{\circledR}$, Probe 407 with PH 07 Miniholder). Exteriorized ileal segment was covered with humidified cotton and gauze, and temperature of gut was maintained with warm water and a heat lamp. After surgical preparation was completed, two large-bore tubes with multiple side-holes were placed in abdominal cavity for later induction of peritonitis, and the laparotomy incision was approached.

\section{Hemodynamics and oxygen utilization}

All intravascular pressure measurements were referenced to midchest level. Cardiac output was measured by thermo-dilution method. Three consecutive determinations of $\mathrm{CO}$ were made by injecting $10 \mathrm{~mL}$ of ice-cold bolus of $5 \%$ dextrose at end expiration. The mean value recorded as $\mathrm{CO}$. Heart rate, MAP, MPAP, PCWP, CVP and core temperature were continuously measured. Systemic pulse pressure variation (PPV) was calculated as the difference of maximal and minimal pulse pressure divided by their mean on one respiratory cycle..$^{13}$ Arterial, mixed venous, and portal vein blood samples were drawn for determinations of blood gases (ABL 520, Radiometer, Copenhagen, Denmark), and for lactate concentration. Hemoglobin concentration $(\mathrm{g} / \mathrm{dL})$ was determined by standard spectrophotometric method. $\mathrm{NaCl}$ concentration was determined by indirect potentiometry (mmol.L 1) and osmolarity (mosmol. $\mathrm{L}^{-1}$ ) was determined by freezing point depression.

\section{Ileal mucosal and muscularis blood flow}

MBF was continuously measured with $\mathrm{LDF}^{14}$ a well-characterized technique for measurement of red blood cell flux in the tissues $^{11,15}$ and because the optical probe has a spatial resolution of $0.5^{-1} \mathrm{~mm} 3$, the obtained measurements reflect only the flow on surface where the probe is applied and not the entire intestinal wall. ${ }^{16}$ LDF signals were exported via analog output and acquired on line via a multichannel interface (Mac Paq MP100; Biopac system Inc., Goleta, CA) with acquisition/analysis software (Acqknowledge 7.2; Biopac system Inc.) to a portable computer. Two different signals are available for external recording. One output is proportional to intensity of backscattered light. This signal is useful to determine whether optical probe is making adequate contact with tissue surface. The second signal is proportional to blood flow, which has been shown to scale linearly with independent measurements of local perfusion in a variety of tissues. LDF are not calibrated to measure absolute blood flow, but indicate MBF in arbitrary perfusion units (PU). Before each experiment, a calibration using random Brownian motion of small scatters in an emulsion (Periflux motility standard, Perimed) was performed. The quality of LDF signal was controlled online by visualizing it on a computer screen, so that motion artefacts and noise due to inadequate probe attachment could be immediately detected and corrected before measurements started. Each reading was the mean of two min of LDF tracing.

\section{Experimental design}

Animals were stabilized for $60 \mathrm{~min}$ before baseline measurements (TB) were performed (Figure 2). Sepsis was induced by injecting

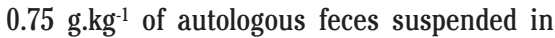
$200 \mathrm{~mL}$ of warm isotonic saline through the abdominal tubes in the peritoneal cavity. After induction of peritonitis, animals received 10 $\mathrm{mL} . \mathrm{kg}^{-1} \cdot \mathrm{h}^{-1}$ of Ringer lactate. Regional and local blood flow was continuously monitored. Systemic hemodynamic variables were meas-

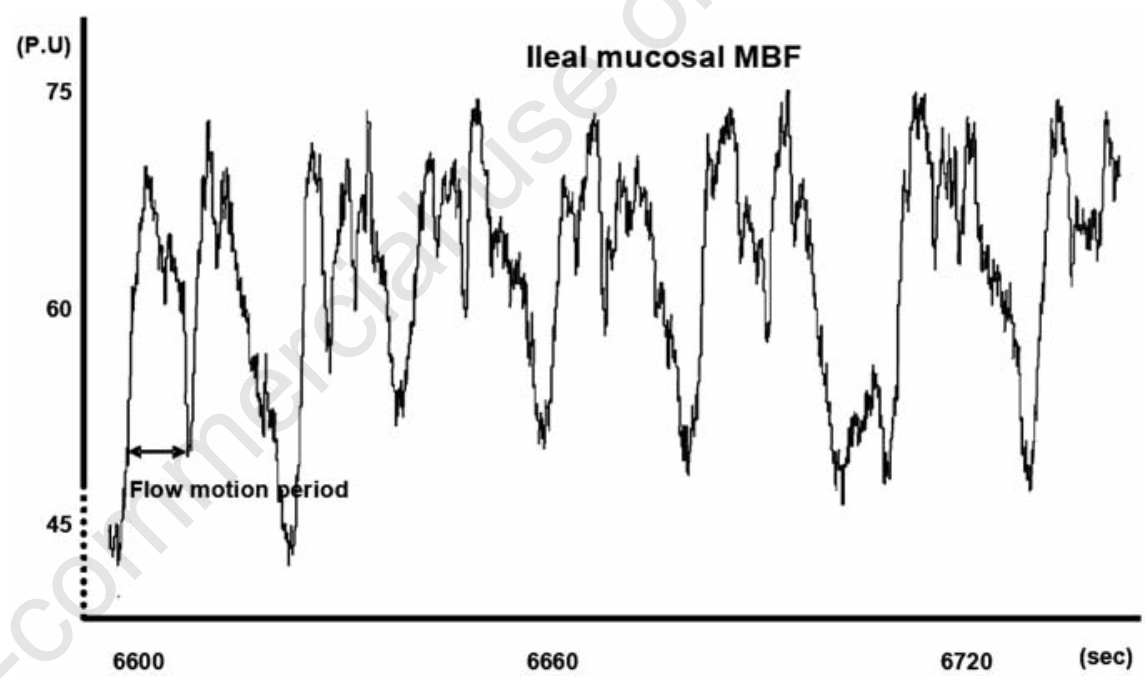

Figure 1. Extracts from 120 seconds of ileal microcirculatory blood flow (MBF) tracing, by LDF in arbitrary perfusion units (PU). Note the absence of respiratory oscillations and peristaltic artefacts. Flow motion oscillations are present with frequency of 4 to 6 per min.

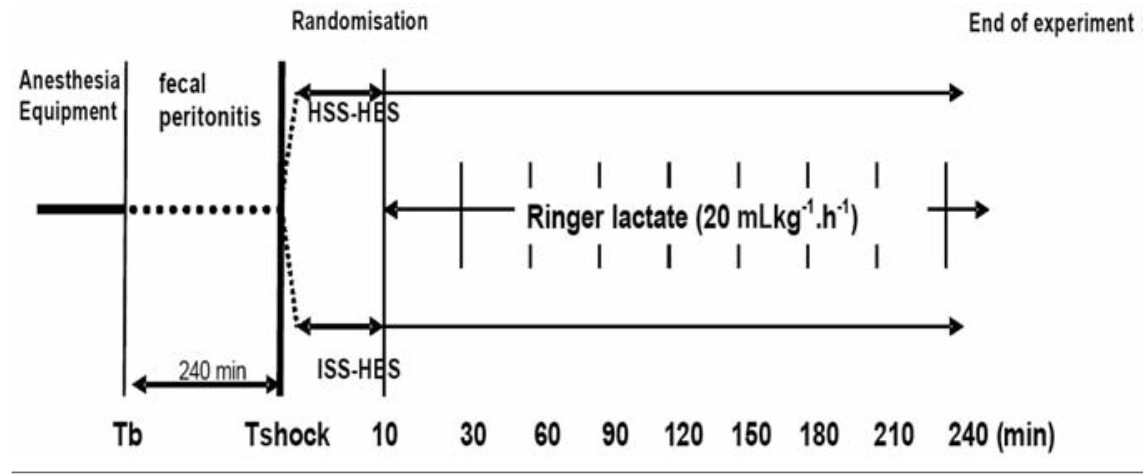

Figure 2. Experimental protocol. After the equipment of pigs, septic shock was induced by fecal peritonitis. Then animals were randomised into two groups to receive equal volumes of either hypertonic saline 7.2\%/hydroxyethyl starch (HSS-HES) or isotonic saline $0.9 \% /$ hydroxyethyl starch (ISS-HES); Animals were observed for $240 \mathrm{~min}$. 
ured hourly. Resuscitation was started at $\mathrm{T}$ shock, when MAP or CI was reduced by $50 \%$ from its baseline (TB) value. They were randomly assigned into two groups just before intervention, thereby minimizing any potential risk of biased handling of animals; the studied group (HSS-HES, $\mathrm{n}=7$ ) received hypertonic saline 7.2\%/6\% hydroxyethyl starch, 200/0.5 (HyperHES ${ }^{\circledR}$, Fresenius Kabi, France) and the control group (ISS-HES, $\mathrm{n}=7$ ) received isotonic saline $0.9 \% / 6 \%$ hydroxyethylstarch, 130/0.4 (VOLUVEN $^{\circledR}$, Fresenius Kabi, France) in raison of $10 \mathrm{~mL} . \mathrm{kg}^{-1}$ infused within $10 \mathrm{~min}$, through a central venous line.

In addition all animals received constant intravenous infusion of $20 \mathrm{~mL} \cdot \mathrm{kg}^{-1} \cdot \mathrm{h}^{-1}$ of Ringer lactate. Microcirculatory blood flow was continuously monitored. Systemic hemodynamic variables were taken at the moment of septic shock (T shock), after $10 \mathrm{~min}$ (T10 min) and halfhourly (T30, T60, T90, T120, T150, T180, T210 and T240 $\mathrm{min}$ ), while blood samples were taken hourly (at T60, T120, T180 and T240 min) during four hours after start of resuscitation.

After completing the experiment, all animals were sacrified with an intravenous injection of potassium chloride $(20 \mathrm{mmol})$ under deep anesthesia.

\section{Statistical analysis}

Because of the large variability of baseline values, measurements of LDF are usually expressed as changes relative to baseline. Data were analyzed using a two-way analysis of variance for repeated measurements followed by a Newman-Keuls procedure. A P value $<0.05$ was considered statistically significant. A multiple linear regression was used to evaluate variations of MBF compared to variations of CI during HSS-HEA infusion. All values are expressed as mean $\pm \mathrm{SD}$, except when mentioned otherwise. We used Sigma Stat 3.1 software for Windows.

\section{Results}

All animals completed the 10-hours study protocol.

\section{The animal peritonitis model}

After 240 min of its induction, fecal peritonitis promoted hemodynamic changes that are characteristic of a hypodynamic septic shock. There were significant $(\mathrm{P}<0.05)$ reductions of MAP, CI, CVP, PCWP and augmentation of PPV. There were severe and significant $(P<0.05)$ stepwise impairment of ileal mucosal MBF ($34 \%)$ and ileal muscularis MBF (-54\%) from baseline (Figure 3). Hemodynamic, ileal microcirculatory blood flow variables during peritonitis are presented in Table 1.

\section{Hemodynamic variables during}

fluid administration

During HSS-HES and ISS-HES fluid administration, CI increased significantly up to min 90 , and then it was progressively decreased. CI under HSS-HES infusion was higher $(\mathrm{P}<0.05)$ up to $\min 30(+37 \%)$. MAP significantly increased up to min 90 during HSS-HES infusion and up to min 30 during ISS-HES infusion with significant difference between the two groups at $30 \mathrm{~min}$ and then MAP progressively deteriorated in both groups. CVP was increased during HSS-HES infusion $(\mathrm{P}<0.05)$ without significant difference between the two groups. There were no significant differences in PPV and PCWP between the two groups. Hemodynamic variables during fluid infusion are presented in Table 2.

\section{Microcirculatory variables during fluid administration}

Ileal mucosal MBF (Figure 4) was significantly augmented after HSS-HES up to min $150(+119 \%)$ compared to shock value, when it was progressively diminished to reach its TB value after 240 min. Meanwhile, Ileal mucosal MBF after ISS-HES insignificantly augmented and approached its $\mathrm{T}$ shock value after 240 min. Mucosal MBF was higher under HSS-HES infusion until the end of the experiment with significant difference between the two groups up to min 120 post shock ( $226 \%$ versus $88 \%$ of shock value). Ileal muscularis MBF were insignificantly increased in both groups during this time and without difference between both groups. The correlation between variation of mucosal MBF and CI was poor $\left(r^{2}=0.2\right.$,

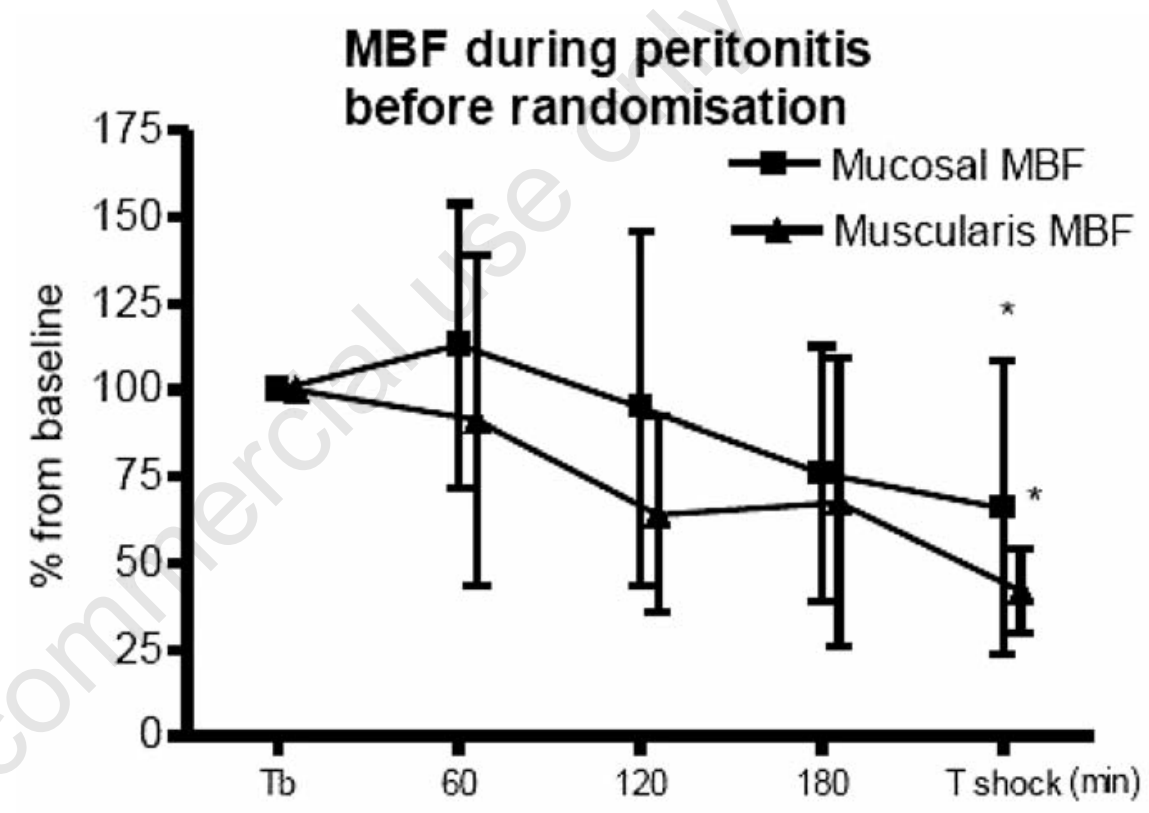

Figure 3. Ileal mucosal and muscularis microcirculatory blood flow (MBF) changes during peritonitis. Peritonitis was induced at TB (baseline). MBF were set at $100 \%$ at TB. Values at $60,120,180$ and 240 (T240=onset of septic shock) min were expressed as percentage of their baseline values. Data are presented as mean \pm SD. ${ }^{*} \mathrm{P}<0.05$ (different from baseline).

Table 1. Hemodynamic data during peritonitis. TB, baseline value before induction of peritonitis. Values were taken at $60,120,180$ and 240 (onset of septic shock) min after induction of peritonitis; MAP, mean arterial pressure; CI, cardiac index; HR, heart rate; CVP, central venous pressure; PCWP, pulmonary capillary wedge pressure; VPP, pulse pressure variation; MPAP, mean pulmonary artery pressure.

\begin{tabular}{lccccc} 
Time (min) & Baseline & T60 & T120 & T180 & T240 \\
MAP (mmHg) & $83 \pm 9$ & $64 \pm 10^{*}$ & $57 \pm 8^{*}$ & $50 \pm 12^{*}$ & $48 \pm 9^{*}$ \\
Cl (ml.kg $\left.{ }^{-1} \mathrm{~min}^{-1}\right)$ & $73 \pm 17$ & $54 \pm 15^{*}$ & $45 \pm 12^{*}$ & $39 \pm 12^{*}$ & $32 \pm 14^{*}$ \\
\hline HR (beats/min) & $95 \pm 13$ & $109 \pm 24^{*}$ & $128 \pm 33^{*}$ & $144 \pm 40^{*}$ & $160 \pm 39^{*}$ \\
CVP (mmHg) & $7 \pm 2$ & $6 \pm 3^{*}$ & $6 \pm 3^{*}$ & $5 \pm 3^{*}$ & $5 \pm 3^{*}$ \\
\hline PCWP (mmHg) & $9 \pm 2$ & $6 \pm 2^{*}$ & $6 \pm 2^{*}$ & $6 \pm 2^{*}$ & $5 \pm 2^{*}$ \\
PPV (\%) & $11 \pm 5$ & $20 \pm 7^{*}$ & $29 \pm 20^{*}$ & $30 \pm 10^{*}$ & $33 \pm 9^{*}$ \\
\hline MPAP (mmg) & $21 \pm 4$ & $20 \pm 5$ & $20 \pm 4$ & $19 \pm 3$ & $19 \pm 3$ \\
\hline D & & & &
\end{tabular}

Data are presented as mean \pm SD. 'P $<0.05$ (significant difference from baseline)

[Infectious Disease Reports 2012; 4:e22]

[page 79] 
Table 2. Hemodynamic variables during fluid administration. After induction of peritonitis, septic shock corresponded to $\mathrm{T}$ shock, when pigs were resuscitated either with (HSS-HES) hypertonic saline $7.2 \%$ /hydroxyethyl starch or (ISS-HES) isotonic saline $0.9 \% /$ hydroxyethyl starch.

\begin{tabular}{|c|c|c|c|c|c|c|c|c|c|c|c|}
\hline Time (min) & & Tshock & T10 & T30 & $\mathrm{T} 60$ & T90 & T120 & T150 & T180 & T210 & $\mathrm{T} 240$ \\
\hline $\begin{array}{l}\text { MAP. } \\
\text { (mmHg) }\end{array}$ & $\begin{array}{l}\text { ISS } \\
\text { HSS }\end{array}$ & $\begin{array}{l}44 \pm 6 \\
40 \pm 9\end{array}$ & $\begin{array}{c}61 \pm 7^{*} \\
67 \pm 13^{*}\end{array}$ & $\begin{array}{c}54 \pm 6^{*} \\
66 \pm 10^{* *}\end{array}$ & $\begin{array}{c}52 \pm 6 \\
56 \pm 6^{*}\end{array}$ & $\begin{array}{c}52 \pm 7 \\
55 \pm 11^{*}\end{array}$ & $\begin{array}{c}49 \pm 10 \\
48 \pm 5\end{array}$ & $\begin{array}{c}49 \pm 10 \\
45 \pm 8\end{array}$ & $\begin{array}{c}47 \pm 12 \\
45 \pm 7\end{array}$ & $\begin{array}{c}46 \pm 12 \\
43 \pm 8\end{array}$ & $\begin{array}{c}46 \pm 13 \\
36 \pm 9\end{array}$ \\
\hline $\begin{array}{l}\mathrm{CI} \\
\left(\mathrm{ml} \mathrm{kg}^{-1} \mathrm{~min}^{-1}\right)\end{array}$ & $\begin{array}{l}\text { ISS } \\
\text { HSS }\end{array}$ & $\begin{array}{l}31 \pm 5 \\
30 \pm 8 \\
\end{array}$ & $\begin{array}{c}51 \pm 15^{*} \\
89 \pm 25^{* \#}\end{array}$ & $\begin{array}{l}53 \pm 14^{*} \\
73 \pm 17^{* \#}\end{array}$ & $\begin{array}{l}49 \pm 13^{*} \\
62 \pm 24^{*}\end{array}$ & $\begin{array}{l}47 \pm 19^{*} \\
55 \pm 24^{*}\end{array}$ & $\begin{array}{l}38 \pm 13 \\
42 \pm 15\end{array}$ & $\begin{array}{l}36 \pm 18 \\
40 \pm 14\end{array}$ & $\begin{array}{l}33 \pm 15 \\
34 \pm 16\end{array}$ & $\begin{array}{l}30 \pm 12 \\
34 \pm 18\end{array}$ & $\begin{array}{l}32 \pm 19 \\
34 \pm 22\end{array}$ \\
\hline $\begin{array}{l}\text { CVP } \\
(\mathrm{mmHg})\end{array}$ & $\begin{array}{l}\text { ISS } \\
\text { HSS }\end{array}$ & $\begin{array}{l}7 \pm 3 \\
4 \pm 2\end{array}$ & $\begin{array}{l}8 \pm 4 \\
9 \pm 3^{*}\end{array}$ & $\begin{array}{l}8 \pm 4 \\
9 \pm 4^{*}\end{array}$ & $\begin{array}{c}8 \pm 3 \\
8 \pm 3^{*}\end{array}$ & $\begin{array}{l}7 \pm 3 \\
7 \pm 3\end{array}$ & $\begin{array}{l}6 \pm 3 \\
5 \pm 2\end{array}$ & $\begin{array}{l}8 \pm 4 \\
6 \pm 3^{*}\end{array}$ & $\begin{array}{l}6 \pm 3 \\
6 \pm 3\end{array}$ & $\begin{array}{c}6 \pm 3 \\
7 \pm 3^{*}\end{array}$ & $\begin{array}{l}6 \pm 2 \\
8 \pm 3^{*}\end{array}$ \\
\hline $\begin{array}{l}\text { PCWP } \\
(\mathrm{mmHg})\end{array}$ & $\begin{array}{l}\text { ISS } \\
\text { HSS }\end{array}$ & $\begin{array}{l}6 \pm 3 \\
5 \pm 2\end{array}$ & $\begin{array}{l}10 \pm 3^{*} \\
10 \pm 2^{*}\end{array}$ & $\begin{array}{c}10 \pm 5^{*} \\
9 \pm 3^{*}\end{array}$ & $\begin{array}{c}9 \pm 3^{*} \\
7 \pm 2\end{array}$ & $\begin{array}{l}8 \pm 3 \\
7 \pm 2\end{array}$ & $\begin{array}{l}7 \pm 4 \\
7 \pm 2\end{array}$ & $\begin{array}{l}7 \pm 3 \\
6 \pm 2\end{array}$ & $\begin{array}{l}8 \pm 2 \\
7 \pm 3\end{array}$ & $\begin{array}{l}7 \pm 2 \\
7 \pm 2\end{array}$ & $\begin{array}{l}8 \pm 2 \\
7 \pm 2\end{array}$ \\
\hline $\begin{array}{l}\text { PPV } \\
(\%)\end{array}$ & $\begin{array}{l}\text { ISS } \\
\text { HSS }\end{array}$ & $\begin{array}{c}29 \pm 12 \\
35 \pm 9\end{array}$ & $\begin{array}{l}14 \pm 4^{*} \\
11 \pm 5^{*}\end{array}$ & $\begin{array}{l}18 \pm 6^{*} \\
15 \pm 5^{*}\end{array}$ & $\begin{array}{c}17 \pm 7 \\
22 \pm 8^{*}\end{array}$ & $\begin{array}{l}20 \pm 11 \\
29 \pm 13\end{array}$ & $\begin{array}{l}26 \pm 13 \\
27 \pm 10\end{array}$ & $\begin{array}{l}25 \pm 8 \\
26 \pm 8\end{array}$ & $\begin{array}{l}21 \pm 5 \\
26 \pm 9\end{array}$ & $\begin{array}{c}19 \pm 2 \\
28 \pm 10\end{array}$ & $\begin{array}{c}22 \pm 6 \\
26 \pm 11\end{array}$ \\
\hline $\begin{array}{l}\text { MPAP } \\
(\mathrm{mmHg})\end{array}$ & $\begin{array}{l}\text { ISS } \\
\text { HSS }\end{array}$ & $\begin{array}{l}20 \pm 3 \\
17 \pm 4\end{array}$ & $\begin{array}{c}28 \pm 3^{* \#} \\
22 \pm 4\end{array}$ & $\begin{array}{l}23 \pm 2 \\
20 \pm 4\end{array}$ & $\begin{array}{c}23 \pm 4^{\#} \\
18 \pm 3\end{array}$ & $\begin{array}{l}23 \pm 4 \\
19 \pm 4\end{array}$ & $\begin{array}{l}24 \pm 2 \\
20 \pm 6\end{array}$ & $\begin{array}{l}24 \pm 5 \\
21 \pm 6\end{array}$ & $\begin{array}{l}25 \pm 5 \\
22 \pm 7\end{array}$ & $\begin{array}{l}25 \pm 5^{*} \\
24 \pm 8^{*}\end{array}$ & $\begin{array}{l}27 \pm 4^{*} \\
24 \pm 7^{*}\end{array}$ \\
\hline
\end{tabular}

MAP, mean arterial pressure; $\mathrm{CI}$, cardiac index; CVP, central venous pressure; PCWP, pulmonary capillary wedge pressure; MPAP, mean pulmonary artery pressure; PPV, pulse pressure variation. Data are presented as mean \pm SD. ${ }^{*} \mathrm{P}<0.05$ (significant difference from T shock). ${ }^{\mathrm{P}}<0.05$ (significant difference between groups).

$\mathrm{P}=0.01)$. The ratio between mucosal MBF and $\mathrm{CI}$ during resuscitation was not different from both groups. Microcirculatory blood flow variables during fluid infusion are presented in Table 2.

\section{Acid-base status during fluid infusion}

Arterial and portal vein lactates did not change significantly over the course of time in ISS-HES and HSS-HES groups and there was no significant difference between the two groups. Arterial $\mathrm{pH}$ and bicarbonates as well as portal vein bicarbonates were within the normal limits at the beginning of resuscitation and stayed as such till the end of the experiment in both groups. Arterial and portal vein $\mathrm{pH}$ did not show significant differences between the two groups. Results are presented in Table 3.

\section{Sodium concentration and}

\section{osmolarity during fluid infusion}

Plasma osmolarity after $180 \mathrm{~min}$ of resuscitation was moderately higher in the HSS-HES group than in the ISS-HES group $(283 \pm 9$ vs. $303 \pm 10, \mathrm{P}<0.05)$ as well as plasma sodium at the end of the experiment $(145 \pm 2$ vs. $134 \pm 3, \mathrm{P}$ $<0.05)$. Results are presented in Table 3 .

\section{Variables of oxygenation during fluid administration}

Oxygen delivery was significantly increased after 60 min in both groups compared to shock values without significant difference between the two groups. Oxygen consumption did not significantly change in both groups. These modifications were accompanied with augmentation of oxygen extraction $(\mathrm{P}<0.05)$ in both groups after 60 and $120 \mathrm{~min}$ of septic shock and without significant difference

Table 3. Acid-base balance. After induction of peritonitis, septic shock took place at T shock, when pigs were resuscitated during $240 \mathrm{~min}$ either with (HSS-HES) hypertonic saline $7.2 \%$ /hydroxyethyl starch or (ISS-HES) isotonic saline $0.9 \% /$ hydroxyethyl starch.

\begin{tabular}{|c|c|c|c|c|c|c|}
\hline Time (min) & & T shock & $\mathrm{T} 60$ & T120 & T180 & $\mathrm{T} 240$ \\
\hline $\begin{array}{l}\text { Arterial lactate } \\
(\mathrm{mmol} / \mathrm{L})\end{array}$ & $\begin{array}{l}\text { ISS } \\
\text { HSS }\end{array}$ & $\begin{array}{l}1.96 \pm 0.57 \\
2.95 \pm 2.21\end{array}$ & $\begin{array}{c}2.26 \pm 0.89 \\
2.90 \pm 2.5\end{array}$ & $\begin{array}{l}2.06 \pm 0.31 \\
2.43 \pm 1.20\end{array}$ & $\begin{array}{l}2.04 \pm 0.52 \\
2.45 \pm 1.52\end{array}$ & $\begin{array}{l}2.62 \pm 1.86 \\
3.60 \pm 2.91\end{array}$ \\
\hline $\begin{array}{l}\text { Portal vein lactate } \\
(\mathrm{mmol} / \mathrm{L})\end{array}$ & $\begin{array}{l}\text { ISS } \\
\text { HSS }\end{array}$ & $\begin{array}{l}2.80 \pm 1.30 \\
3.92 \pm 2.32\end{array}$ & $\begin{array}{l}2.86 \pm 1.16 \\
2.41 \pm 0.63\end{array}$ & $\begin{array}{l}2.94 \pm 1.20 \\
2.46 \pm 0.95\end{array}$ & $\begin{array}{l}2.93 \pm 1.22 \\
2.09 \pm 0.89\end{array}$ & $\begin{array}{l}4.54 \pm 2.64 \\
3.33 \pm 1.51\end{array}$ \\
\hline $\begin{array}{l}\text { Arterial bicarbonates } \\
(\mathrm{mmol} / \mathrm{L})\end{array}$ & $\begin{array}{l}\text { ISS } \\
\text { HSS }\end{array}$ & $\begin{array}{l}38 \pm 3 \\
41 \pm 6\end{array}$ & $\begin{array}{l}39 \pm 4 \\
40 \pm 9\end{array}$ & $\begin{array}{l}41 \pm 6 \\
39 \pm 7\end{array}$ & $\begin{array}{l}37 \pm 6 \\
41 \pm 5\end{array}$ & $\begin{array}{l}38 \pm 6 \\
42 \pm 5\end{array}$ \\
\hline $\begin{array}{l}\text { Portal vein bicarbonates } \\
(\mathrm{mmol} / \mathrm{L})\end{array}$ & $\begin{array}{l}\text { ISS } \\
\text { HSS }\end{array}$ & $\begin{array}{l}30 \pm 2 \\
27 \pm 2\end{array}$ & $\begin{array}{l}27 \pm 3 \\
26 \pm 2\end{array}$ & $\begin{array}{l}26 \pm 4 \\
27 \pm 3\end{array}$ & $\begin{array}{l}28 \pm 2 \\
26 \pm 3\end{array}$ & $\begin{array}{l}26 \pm 4 \\
24 \pm 4\end{array}$ \\
\hline Arterial pH & $\begin{array}{l}\text { ISS } \\
\text { HSS }\end{array}$ & $\begin{array}{l}7.44 \pm 0.03 \\
7.38 \pm 0.05\end{array}$ & $\begin{array}{l}7.44 \pm 0.03 \\
7.36 \pm 0.07\end{array}$ & $\begin{array}{l}7.42 \pm 0.04 \\
7.39 \pm 0.06\end{array}$ & $\begin{array}{l}7.43 \pm 0.05 \\
738 \pm 0.04\end{array}$ & $\begin{array}{l}7.41 \pm 0.03 \\
7.35 \pm 0.08\end{array}$ \\
\hline Portal venous pH & $\begin{array}{l}\text { ISS } \\
\text { HSS }\end{array}$ & $\begin{array}{l}7.35 \pm 0.04 \\
7.27 \pm 0.03\end{array}$ & $\begin{array}{l}7.39 \pm 0.03 \\
7.33 \pm 0.04\end{array}$ & $\begin{array}{l}7.37 \pm 0.03 \\
7.26 \pm 0.03\end{array}$ & $\begin{array}{l}7.37 \pm 0.04 \\
7.28 \pm 0.01\end{array}$ & $\begin{array}{l}7.32 \pm 0.07 \\
7.30 \pm 0.05\end{array}$ \\
\hline $\begin{array}{l}\text { Sodium } \\
\left(\mathrm{mmol.L^{-1 }}\right)\end{array}$ & $\begin{array}{l}\text { ISS } \\
\text { HSS }\end{array}$ & $\begin{array}{l}135 \pm 4 \\
135 \pm 3\end{array}$ & $\begin{array}{c}135 \pm 2 \\
149 \pm 4^{* \#}\end{array}$ & $\begin{array}{c}135 \pm 2 \\
148 \pm 4^{* \sharp}\end{array}$ & $\begin{array}{c}134 \pm 2 \\
146 \pm 3^{* \#}\end{array}$ & $\begin{array}{c}134 \pm 3 \\
145 \pm 2^{* \#}\end{array}$ \\
\hline $\begin{array}{l}\text { Osmolarity } \\
\left(\text { mosmol. } L^{-1}\right)\end{array}$ & $\begin{array}{l}\text { ISS } \\
\text { HSS }\end{array}$ & $\begin{array}{l}284 \pm 6 \\
284 \pm 6\end{array}$ & $\begin{array}{c}285 \pm 5 \\
311 \pm 7^{*}\end{array}$ & $\begin{array}{c}285 \pm 7 \\
292 \pm 37\end{array}$ & $\begin{array}{c}283 \pm 6 \\
303 \pm 10^{* / \#}\end{array}$ & $\begin{array}{c}283 \pm 9 \\
270 \pm 83\end{array}$ \\
\hline
\end{tabular}

Data are presented as mean $\pm \mathrm{SD}$. " $\mathrm{P}<0.05$ (significant difference from $\mathrm{T}$ shock). ${ }^{*} \mathrm{P}<0.05$ (significant difference between groups).

between the two groups. Mixed venous oxygen saturation $\left(\mathrm{SmvO}_{2}\right)$ was augmented $(\mathrm{P}<0.05)$ in both groups after 120 min without difference between the groups. Portal vein oxygen saturation $\left(\mathrm{SpvO}_{2}\right)$ was augmented significantly during ISS-HES infusion compared to shock value. Hemoglobin concentration was stable in both groups and there was no difference between groups. Hemoglobin and variables of oxygenation are shown in Table 4 .

\section{Discussion}

Our main finding in this study is that smallvolume resuscitation with HSS-HES improved ileal mucosal microcirculatory impairment after septic shock caused by experimental peritonitis to find its baseline (TB) values at the end of the experiment (Figure 5). On the contrary, resuscitation with equal volume of ISSHES, despite an initial non-significant improvement, mucosal MBF decreased progressively below shock values. In fact, HSSHES-treated animals have approximately doubled the Doppler signal compared to ISS-HES.

Interestingly, during HSS-HES infusion, improvement of mucosal MBF was maintained till the end of the experiment although MAP and $\mathrm{CI}$ were only preserved up to min 90 . These beneficial effects on macrocirculation have not been confirmed on non septic animals. ${ }^{17}$ In our study, CVP was also better preserved with HSSHES than with ISS-HES, probably due to mobilization of fluids from intracellular to extracel- 
lular compartment by the osmotic gradient produced by HSS-HES. Moreover, the correlation between MBF and systemic hemodynamic was very weak $\left(r^{2}=0.2\right.$ for $\left.\mathrm{CI}\right)$. For the rest, Figure 5 shows a tendency of a better amelioration of MBF than CI during resuscitation with HSSHES than with ISS-HES. We can then postulate that HSS-HES have a specific microcirculatory effect. Similarly, Zakaria et al. ${ }^{18}$ demonstrated this effect by using an in vivo videomicroscopy and optical Doppler velocimetry on intestinal villi in a hemorrhagic shock model. They found that hypertonic saline resuscitation improves intestinal perfusion by selective vasodilatation of precapillary arterioles even at MAP close to shock levels. In accordance to our study, this vasodilatation may also occur during HSS-HES infusion. This demonstrates the interest of using hypertonic saline resuscitation during the early phase of severe sepsis and septic shock to combat tissue ischemia even if MAP is still low.

Our data confirm a study showing that under a combination of hypertonic saline (7.5\%) with a dextran, MBF was augmented during the first two hours of resuscitation compared to an association of isotonic saline plus a dextran. ${ }^{15}$ However, authors used a model of (endotoxemia) septic shock which is more averted from human septic shock than ours (experimental peritonitis). Moreover, they used another colloid and did not study the correlation between variations of systemic output and mucosal MBF. Somell et al. showed an improvement of survival in a swine endotoxemia model under a combination of hypertonic saline (7.5\%) with a dextran, but authors did not study any microcirculatory effect. ${ }^{19}$ In their study, they used isotonic saline as control, therefore making it difficult to separate the beneficial effect of either HSS or colloid.

During peritonitis (before resuscitation), there were different reductions of microcirculatory blood flow in the ileal mucosa $(-34 \%)$ and ileal muscularis (-59\%). It seems possible that blood flow was deviated from muscularis towards the mucosa during peritonitis which had reached its maximum at the onset of shock. On the other hand, after resuscitation with HSS-HES, there was a higher augmentation of MBF in the mucosa than in the muscularis. These blood flow modifications can be explained by the redistribution of blood flow between ileal mucosa and muscularis during shock and after resuscitation for the same. This muscularis ischemia might be responsible for paralytic ileus observed in septic shock. Contrary to our study, Revelly et al. ${ }^{20}$ had observed an augmentation of mucosal MBF during endotoxic shock possibly because of intensive fluid resuscitation.

Among the ISS-HES group, portal vein oxygen saturation $\left(\mathrm{SO}_{2}\right)$ was significantly augmented. This might be due to the phenomenon
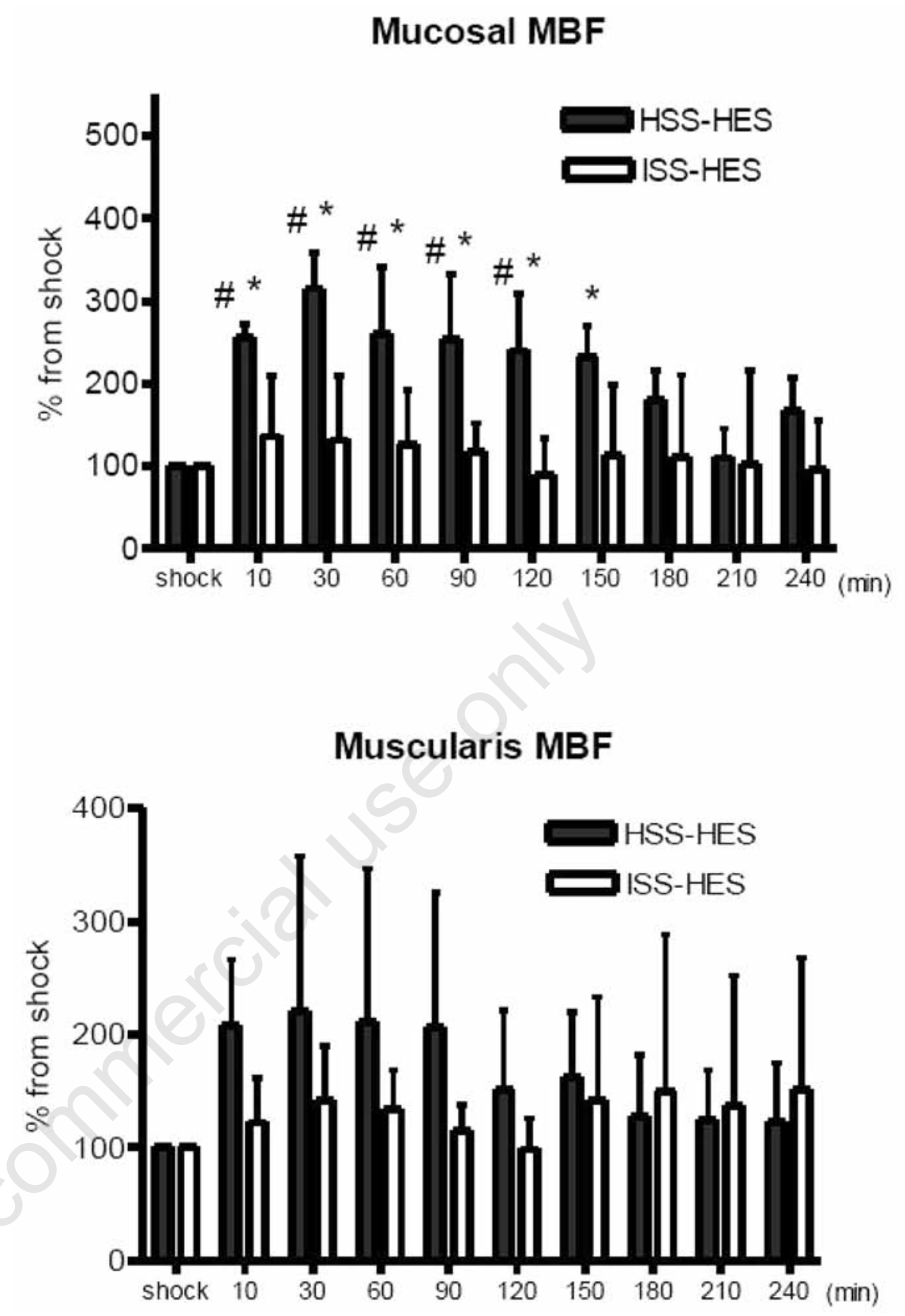

Figure 4. Relative ileal mucosal and muscularis microcirculatory blood flow changes during fluid administration after the onset of septic shock. Pigs were resuscitated with either hypertonic saline $7.2 \%$ / hydroxyethyl starch (HSS-HES) or isotonic saline $0.9 \%$ /hydroxyethyl starch (ISS-HES). All microcirculatory blood flows (MBF) were set at $100 \%$ before fluid administration at $T$ shock and measured values were expressed as percentage of $T$ shock. Data are presented as mean $\pm S D .{ }^{*} P<0.05$ significant difference from $T$ shock; ${ }^{\#} P$ $<0.05$ significant difference between groups.

of capillary shunting, ${ }^{21}$ in which blood had shunted from precapillary arterioles to postcapillary venules without passing through the tissue capillaries. This shunting might have been caused by lack of proper precapillary dilatation during ISS-HES infusion contrary to HSS-HES infusion when portal vein $\mathrm{SO}_{2}$ was not augmented. For the same reason, while portal vein lactate was slightly augmented during ISS-HES infusion, portal vein lactate was slightly improved till T180 min during HSS-
HES infusion (Table 3). All these modifications during HSS-HES infusion might be explained by the augmentation of splanchnic MBF, regional blood flow redistribution toward mucosal tissue and/or due to an effect at cellular level that enables tissues to increase their oxygen extraction as oxygen supply is lowered.

During the current study, plasma osmolarity and sodium levels were increased significantly with a single infusion of hypertonic saline solution and this effect lasted during the whole 
duration of the experiment. The potential risk of inducing important hypertonic states with possible harmful consequences to patients must be kept in mind and looked into, particularly after repetitive infusions of hypertonic saline solutions. ${ }^{22}$

One advantage of this study is the reproducibility of its results. In this regard, during the whole duration of this experiment, we were able to measure ileal MBF by LDF continuously at one precised point by fixing the exposed ileal mucosa on a platform (see materials and methods); so that any artefact caused by respiratory and peristaltic movements was eliminated. Hemoglobin was stable during the whole duration of the experiment, therefore avoiding any false readings resulting from modification of red blood cell count, as the LDF probe detects movement of red blood cells in relation to fixed tissues. Likewise, animals were resuscitated in the same manner, thereby avoiding any bias resulting from fluid loading difference between the two groups.

An important limit of this study is the exteriorisation of the ileum. Despite the precautions that were taken to keep the exposed intestine moist and warm, this approach may be less physiological. Nevertheless, these LDF tracings were reproducible and reliable. Furthermore, the LDF tracings were quite physiological with rhythmical oscillations (vasomotion) and these became more obvious after onset of sepsis (Extract of LDF recording Figure 1). Nevertheless, LDF measures a small volume of $\mathrm{MBF}$ (one $\mathrm{mm}^{3}$ approximately) which includes arterioles, capillaries and veinules. Therefore we were not able to distinguish potential variations of microcirculation heterogeneity between both groups.

Another apparent limit is that animals were not fully resuscitated, despite oxygen extraction ratio $\left(\mathrm{O}_{2}\right.$ ER) was augmented 60 min after resuscitation in both groups. However, our aim was to show if there might be a significant difference or not between the two groups (ISSHES and HSS-HES) by utilizing equal volumes of fluid. Furthermore as this experiment was done to study a physiologic effect; accordingly, hemodynamic results were the consequences rather than the end points of resuscitation. In this way, we can easily judge if a significant difference is due to the type of fluid rather than to its quantity.

Other possible limits of our study are the small number of animals and the short observation time. Consequently, it was impossible to analyze the impact of fluid resuscitation on the development of multiple organ dysfunction and survival. Generally speaking, endotoxemia models present often a shorter survival; ${ }^{19}$ therefore affording a worse survival analysis.

Although the potential beneficial effect of small volume resuscitation with hypertonic solutions has been extensively studied in hypovolemic shock, but animal and clinical studies that have been directed at treatment of septic shock using hypertonic solutions are limited. A chronic model to evaluate the potential beneficial effects of hypertonic saline solutions during longer period, in addition to its utilization during the early stages of septic shock has not been investigated. Recently in their review, Oliveira et al. commented on the mechanisms of action of hypertonic saline and discussed the use of hypertonic solutions for treatment of septic shock and they pointed out the potential benefits of using hypertonic solu- tions in patients with sepsis. ${ }^{9}$ These improvements following hypertonic saline resuscitation might have a greater impact on the subsequent course of patients admitted to the intensive care unit. Indeed, Rivers et al. ${ }^{23}$ showed that early goal-directed fluid resuscitation in patients with septic shock provides significant benefits related to outcome in the first 6 hours after admission to the emergency department. Our findings can be exploited in this sense with the utilization of HSS-HES during early resuscitation to augment microcirculatory blood flow and to improve tissue oxygenation.

Table 4. Variables of oxygenation during fluid administration. After induction of peritonitis, septic shock took place at $\mathrm{T}$ shock, when pigs were resuscitated during $\mathbf{2 4 0}$ min either with (HSS-HES) hypertonic saline 7.2\%/ hydroxyethyl starch or (ISS-HES) isotonic saline $0.9 \% / h y d r o x y e t h y l$ starch. $\mathrm{DO}_{2}=$ oxygen delivery, $\mathrm{VO}_{2}=$ mesenteric oxygen consumption, $\mathrm{ER}=$ oxygen extraction, $\mathrm{SO}_{2}=$ oxygen saturation, $\mathrm{CI}=$ cardiac index.

\begin{tabular}{|c|c|c|c|c|c|c|}
\hline Time (min) & & T shock & T60 & T120 & T180 & $\mathrm{T} 240$ \\
\hline $\begin{array}{l}\text { Mixed venous- } \mathrm{SO}_{2} \\
(\%)\end{array}$ & $\begin{array}{l}\text { ISS } \\
\text { HSS }\end{array}$ & $\begin{array}{l}54 \pm 12 \\
55 \pm 13\end{array}$ & $\begin{array}{l}67 \pm 8^{*} \\
76 \pm 4^{*}\end{array}$ & $\begin{array}{c}66 \pm 11^{*} \\
68 \pm 8\end{array}$ & $\begin{array}{c}60 \pm 10 \\
64 \pm 8\end{array}$ & $\begin{array}{l}56 \pm 16 \\
56 \pm 17\end{array}$ \\
\hline $\begin{array}{l}\text { Portal vein- } \mathrm{SO}_{2} \\
(\%)\end{array}$ & $\begin{array}{l}\text { ISS } \\
\text { HSS }\end{array}$ & $\begin{array}{l}60 \pm 7 \\
71 \pm 9\end{array}$ & $\begin{array}{l}79 \pm 6^{*} \\
83 \pm 9\end{array}$ & $\begin{array}{c}77 \pm 11^{*} \\
68 \pm 5\end{array}$ & $\begin{array}{c}68 \pm 18 \\
67 \pm 8\end{array}$ & $\begin{array}{l}77 \pm 11 \\
71 \pm 16 \\
\end{array}$ \\
\hline $\begin{array}{l}\mathrm{DO}_{2} \\
\left(\mathrm{~mL} \cdot \mathrm{min}^{-1} \cdot \mathrm{m}^{-2}\right)\end{array}$ & $\begin{array}{l}\text { ISS } \\
\text { HSS }\end{array}$ & $\begin{array}{l}4.77 \pm 1.07 \\
4.16 \pm 1.40\end{array}$ & $\begin{array}{l}6.56 \pm 1.82^{*} \\
8.40 \pm 0.10^{*}\end{array}$ & $\begin{array}{l}5.33 \pm 1.33 \\
7.26 \pm 1.58\end{array}$ & $\begin{array}{l}4.63 \pm 2.33 \\
5.62 \pm 0.98\end{array}$ & $\begin{array}{l}4.58 \pm 2.33 \\
4.13 \pm 3.51\end{array}$ \\
\hline $\begin{array}{l}\mathrm{VO}_{2} \\
\left(\mathrm{~mL} \cdot \mathrm{min}^{-1} \cdot \mathrm{m}^{-2}\right)\end{array}$ & $\begin{array}{l}\text { ISS } \\
\text { HSS }\end{array}$ & $\begin{array}{l}2.54 \pm 0.18 \\
1.60 \pm 0.41\end{array}$ & $\begin{array}{l}2.20 \pm 0.96 \\
1.55 \pm 0.22\end{array}$ & $\begin{array}{l}1.91 \pm 0.83 \\
1.91 \pm 0.29\end{array}$ & $\begin{array}{l}1.89 \pm 1.00 \\
1.96 \pm 0.43\end{array}$ & $\begin{array}{l}1.87 \pm 0.66 \\
1.36 \pm 0.25\end{array}$ \\
\hline $\begin{array}{l}\mathrm{O}_{2} \mathrm{ER} \\
(\%)\end{array}$ & $\begin{array}{l}\text { ISS } \\
\text { HSS }\end{array}$ & $\begin{array}{l}0.51 \pm 0.22 \\
0.43 \pm 0.12\end{array}$ & $\begin{array}{l}0.32 \pm 0.09^{*} \\
0.22 \pm 0.03^{*}\end{array}$ & $\begin{array}{l}0.33 \pm 0.11^{*} \\
0.29 \pm 0.08^{*}\end{array}$ & $\begin{array}{l}0.40 \pm 0.10 \\
0.35 \pm 0.09\end{array}$ & $\begin{array}{l}0.43 \pm 0.16 \\
0.43 \pm 0.20\end{array}$ \\
\hline $\mathrm{CI} / \mathrm{O}_{2} \mathrm{ER}$ & $\begin{array}{l}\text { ISS } \\
\text { HSS }\end{array}$ & $\begin{array}{l}2 \pm 1 \\
2 \pm 1\end{array}$ & $\begin{array}{l}4 \pm 2^{*} \\
7 \pm 3^{*}\end{array}$ & $\begin{array}{l}4 \pm 2^{*} \\
4 \pm 2^{*}\end{array}$ & $\begin{array}{l}2 \pm 1 \\
2 \pm 1\end{array}$ & $\begin{array}{l}2 \pm 2 \\
2 \pm 2\end{array}$ \\
\hline $\begin{array}{l}\text { Hemoglobin } \\
(\mathrm{g} / \mathrm{dL})\end{array}$ & $\begin{array}{l}\text { ISS } \\
\text { HSS }\end{array}$ & $\begin{array}{c}11 \pm 1 \\
11 \pm 1.6\end{array}$ & $\begin{array}{l}9.5 \pm 1.6^{*} \\
9.1 \pm 0.8^{*}\end{array}$ & $\begin{array}{l}9.6 \pm 1.6^{*} \\
9.6 \pm 0.7^{*}\end{array}$ & $\begin{array}{c}9.5 \pm 2^{*} \\
9.6 \pm 1.5^{*}\end{array}$ & $\begin{array}{l}9.9 \pm 1.9^{*} \\
9.6 \pm 1.5^{*}\end{array}$ \\
\hline
\end{tabular}

Data are presented as mean \pm SD. ${ }^{*} \mathrm{P}<0.05$ (significant difference from $\mathrm{T}$ shock). ${ }^{*} \mathrm{P}<0.05$ (significant difference between groups).

\section{Mucosal MBF as fraction of cardiac index}

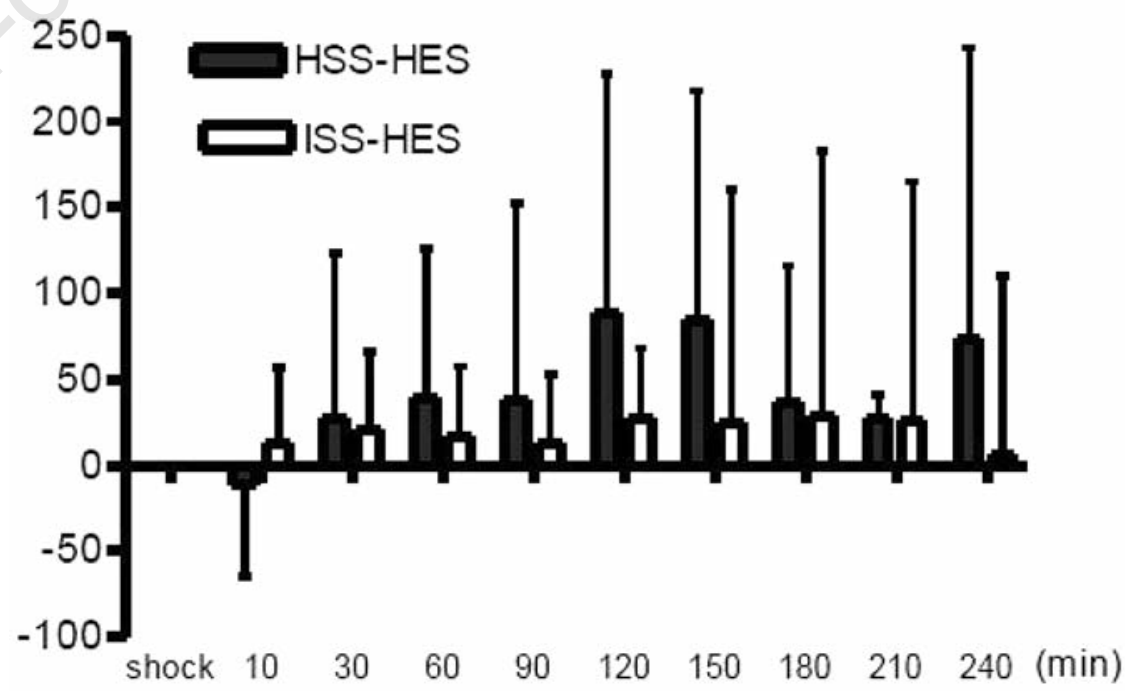

Figure 5. Changes in mucosal microcirculatory blood flows (MBF) compared with changes in cardiac index during fluid administration after the onset of septic shock. Pigs were resuscitated with hypertonic saline $7.2 \%$ /hydroxyethyl starch (HSS-HES) or isotonic saline $0.9 \% /$ hydroxyethyl starch (ISS-HES). The ratio between mucosal MBF and cardiac index at shock was set at 0 . Data are presented as mean \pm SD 


\section{Conclusions}

Resuscitation with HSS-HES during early phase of severe sepsis, compared with ISSHES, augmented ileal mucosal microcirculatory blood flow even when MAP was low. Muscularis MBF was not augmented, maybe by redistribution of flow towards mucosa. The potential long-term beneficial effects of early infusions of HSS-HES during the course of chronic model of severe sepsis need further studies, including the potential impact on the mortality and the possible risk of induced hypertonic states.

\section{References}

1. Garrido AG, Poli de Figueiredo LF, Cruz Jr RJ, et al. Short-lasting systemic and regional benefits of early crystalloid infusion after intravenous inoculation of dogs with live Escherichia coli. Braz J Med Biol Res 2005;38:873-84.

2. Velasco IT, Pontieri V, Rocha e Silva M Jr, et al. Hyperosmotic $\mathrm{NaCl}$ and severe hemorrhagic shock. Am J Physiol 1980;239:H66473.

3. Schmall LM, Muir WW, Robertson JT. Haemodynamic effects of small volume hypertonic saline in experimentally induced haemorrhagic shock. Equine Vet $\mathrm{J}$ 1990;22:273-7.

4. Gurfinkel V, Poggetti RS, Fontes B, et al. Hypertonic saline improves tissue oxygenation and reduces systemic and pulmonary inflammatory response caused by hemorrhagic shock. J Trauma 2003;54:1137-45.

5. Pascual JL, Khwaja KA, Ferri LE, et al. Hypertonic saline resuscitation attenuates neutrophil lung sequestration and trans- migration by diminishing leukocyteendothelial interactions in a two-hit model of hemorrhagic shock and infection. $\mathrm{J}$ Trauma 2003;54:121-30;discussion 30-2.

6. Pascual JL, Ferri LE, Seely AJ, et al. Hypertonic saline resuscitation of hemorrhagic shock diminishes neutrophil rolling and adherence to endothelium and reduces in vivo vascular leakage. Ann Surg 2002;236:634-42.

7. Shields CJ, O'Sullivan AW, Wang JH, et al. Hypertonic saline enhances host response to bacterial challenge by augmenting receptor-independent neutrophil intracellular superoxide formation. Ann Surg 2003;238:249-57.

8. Loomis WH, Namiki S, Hoyt DB, et al. Hypertonicity rescues $\mathrm{T}$ cells from suppression by trauma-induced anti-inflammatory mediators. Am J Physiol Cell Physiol 2001;281:C840-8.

9. Oliveira RP, Velasco I, Soriano F et al. Clinical review: hypertonic saline resuscitation in sepsis. Crit Care 2002;6:418-23.

10. Kramer GC, Wade CE, Prough DS. Hypertonic saline dextran: efficacy and regulatory approval. Acta Anaesthesiol Scand 1998;42:141-4.

11. Hiltebrand LB, Krejci V, Banic A, et al. Dynamic study of the distribution of microcirculatory blood flow in multiple splanchnic organs in septic shock. Crit Care Med 2000;28:3233-41.

12. Lagoa CE, de Figueiredo LF, Cruz RJ Jr. et al. Effects of volume resuscitation on splanchnic perfusion in canine model of severe sepsis induced by live Escherichia coli infusion. Crit Care 2004;8:R221-8.

13. Michard F, Boussat S, Chemla D, et al. Relation between respiratory changes in arterial pulse pressure and fluid responsiveness in septic patients with acute circulatory failure. Am J Respir Crit Care Med
2000;162:134-8.

14. Shepherd AP, Riedel GL: Continuous measurement of intestinal mucosal blood flow by laser-Doppler velocimetry. Am J Physiol 1982; 242:G668-72.

15. Oi Y, Aneman A, Svensson M, et al. Hypertonic saline-dextran improves intestinal perfusion and survival in porcine endotoxin shock. Crit Care Med 2000;28: 2843-50.

16. Kiel JW, Riedel GL, DiResta GR, et al. Gastric mucosal blood flow measured by laser-Doppler velocimetry. Am J Physiol 1985;249:G539-45.

17. Wan L, Bellomo R, May CN. The effects of normal and hypertonic saline on regional blood flow and oxygen delivery. Anesth Analg 2007;105:141-7.

18. Zakaria el R, Tsakadze NL, Garrison RN. Hypertonic saline resuscitation improves intestinal microcirculation in a rat model of hemorrhagic shock. Surgery 2006;140: 579-87; discussion 87-8.

19. Somell A, Sollevi A, Suneson A, et al. Beneficial effects of hypertonic saline/dextran on early survival in porcine endotoxin shock. Acta Anaesthesiol Scand 2005;49: 1124-34.

20. Revelly JP, Ayuse T, Brienza N, et al. Endotoxic shock alters distribution of blood flow within the intestinal wall. Crit Care Med 1996;24:1345-51.

21. Ince C, Sinaasappel M. Microcirculatory oxygenation and shunting in sepsis and shock. Crit Care Med 1999;27:1369-77.

22. Vassar MJ, Perry CA, Holcroft JW. Analysis of potential risks associated with 7.5\% sodium chloride resuscitation of traumatic shock. Arch Surg 1990;125:1309-15.

23. Rivers E, Nguyen B, Havstad S, et al. Early goal-directed therapy in the treatment of severe sepsis and septic shock. N Engl J Med 2001;345:1368-77. 